\title{
Nerveless Spider Genitalia and Other Evolutionary Wonders
}

\author{
The Evolution of Primary Sexual Characters in Animals, by Janet L. Leonard \\ and Alex Córdoba-Aguilar (eds.). Oxford: Oxford University Press, \\ 2010. Pp. v + 537. H/b $\$ 79.95$
}

\author{
Rachael A. Carmen • Haley M. Dillon • Glenn Geher
}

Published online: 16 February 2011

(C) Springer Science+Business Media, LLC 2011

"One of the great joys of biology is the realization that there is no end of wonders to discover, to describe, and to attempt to understand" (p. 1).

Sexual characteristics vary vastly across species, from shape to size to function. The extent to which genital characters vary is obvious when considering the nature of genitalia across the gamut of spider species. Different from other classes of animals, the male spider's genitalia is fully devoid of nerves. The mere idea of nerveless genitalia would shock and appall most sexually active individuals, yet, the chapters that make up The Evolution of Primary Sexual Characters in Animals show that our preconceptions and notions on our sexual organs do not necessarily apply across species.

The Evolution of Primary Sexual Characters in Animals, edited by Janet L. Leonard and Alex Córdoba-Aguilar, is an extensive collection of essays dedicated to providing the reader with an understanding of evolution's impact on the sexual/reproductive aspects of the animal kingdom. The first few chapters give the reader a general overview of evolutionary theory-an exceptional bridge to the rest of the book, which is devoted to the primary sex characters of selected taxa-ranging from marine invertebrates to mammals. The span of creatures varies widely, providing the reader with a cohesive body of knowledge of how various species' genitalia are thought to have evolved. Above and

\footnotetext{
R. A. Carmen $(\varangle) \cdot$ H. M. Dillon $\cdot$ G. Geher

The State University of New York at New Paltz,

JFT 314 (Psychology Department),

1 Hawk Drive,

New Paltz, NY 12561, USA

e-mail: Carmen42@newpaltz.edu
}

beyond the plethora of evolutionary-themed facts, the 22 essays blend into one another as an introductory work to the complexities of sexual characteristics across a colorful array of varying species. Because the book is so extensive, it seemed almost impossible to give each contributing author his or her turn in the limelight, so before proceeding, it must be noted that each chapter is unique and engaging in its own way, and that anyone curious about these specialized subjects should definitely read this book. That said, a few of the chapters proved to be particularly useful to the study of evolution in a general sense. Coming from a research psychology background with a concentration in evolutionary studies and human sexuality (as the authors of this paper do), the beginning and ending chapters proved to be most beneficial from a general evolutionary educational point of view.

The introduction, entitled "Celebrating and Understanding Reproductive Diversity" was written by one of the editors, Janet Leonard, and is packed with extremely useful information regarding the basic principles that would later be reviewed in the book. The general goal is to show the readers a plethora of bizarre reproductive adaptations that are seemingly only known to taxonomists, but the reader soon learns that there is more to the book than just interesting facts regarding differing reproductive adaptations. The information in this chapter provides an excellent preface to the principles and history of evolutionary theory. According to Leonard, since Darwin's time, biologists have pretty much understood that all traits are essentially reproductive traits in some way or another. This thought alone is a great focal point for educators. Though reproduction is always talked about when discussing evolutionary theory, it should more often be discussed as a core element of evolution: "survival and growth are only 
important in so far as they enhance an organism's lifetime reproductive success, so that the adaptive value of traits that serve in foraging, predator evasion, temperature adaptation, resistance to desiccation, etc., comes from their effect on reproduction" (p. 2). By boiling evolutionary theory down to its basics, it is clear that everything in life revolves around reproduction.

Michael Ghiselin's chapter, "The Distinction between Primary and Secondary Sexual Characters," is dedicated to explicating the evolution of basic sexual characters. Per this chapter, in general, a sexual character can mean a part (penis or spine) or it can be an attribute of a part (if we say that the spine is sharp). When one factors in the differing types of selection, the divergence between primary and secondary sexual characters can be easily differentiated: Darwin explained differences between the sexes through natural and sexual selection. Natural selection causes primary sexual characters and sexual selection causes secondary sexual characters. From an educational standpoint, the most useful aspect of this chapter in particular is the information on the difference between natural, sexual, and artificial selection. "All three work as a result of differential reproduction of the components of populations" (this can mean organisms, families, or even differential reproduction of gametes) (p. 10). The difference between the three has to do with the "selective agent" (that is at work within a given population) that causes individuals to have differential reproductive success: in artificial selection the agent is the breeder; in sexual selection, usually the female; in natural selection, the environment. Once again, everything can be boiled down to reproductive success. The author of this essay provides the reader with a tremendously useful way to remember the disparity between differing types of selection. Consider a population that is under carrying capacity. If a selective agent has a positive influence on the population as a whole, it is natural selection. If a selective agent has a negative influence on the population as a whole (or favors individual differences), it is sexual selection. Of course, it isn't as simple as that. A selective agent refers to an individual making a choice (in the case of artificial selection, the breeder; in sexual selection, the member of the opposite sex); so technically natural selection has no true agent (in this case, it is more about which organism makes more effective use of the environment in relation to other organisms). This chapter is also filled with information regarding the different ways that sexual selection operates. On one hand, sexual selection can be carried out through male combat (dominance wins the female), and on the other, it can be carried out through female choice (the most attractive wins the female). Overall, this chapter was by far the most useful when it came to evolutionary theories - a college student who reads this chapter should have a solid understanding of basic evolutionary principles.
Another chapter by Janet Leonard ("The Evolution of Sexes, Anisogamy, and Sexual Systems") goes over some key biology terms in relation to reproduction. In particular, this chapter is about the advantage of having two mating types. According to Leonard, multiple mating types (i.e., more than two) are advantageous if time is of the essence. As the number of mating types increases, so too does the likelihood of successfully having offspring from a random encounter in nature. However, it is rare to hear of multiple mating types. The advantage of having only two matingtype alleles is most likely associated with the competitive advantage to the carrier itself and thus, multiple mating types are not favored. "That is, if a need for rapid zygote formation does not give a big advantage to multiple mating types, natural selection and genetic drift will tend to drive the system to the absolute minimum number of mating types, just two, assuming that outcrossing is beneficial" (p. 17). Overall, Janet Leonard does a great job at answering general biological questions in regard to sexual reproduction.

The chapter by William G. Eberhard entitled "Rapid Divergent Evolution of Genitalia" discusses the recent developments in several fields regarding the link between genital evolution and sexual selection. Other than explaining the influence of non-genital contact structures (or secondary genitalia) on sexual reproduction, the most important information in this chapter contains competing hypotheses regarding the rapid divergent evolution of genitalia. "The Lock and Key" is a somewhat dated hypothesis and is currently being refuted, but it states that selection on females (to avoid insemination by males of other species) has caused female genital structures that "prevent entry or coupling by the male genitalia of other species" (p. 46). In addition to older hypotheses, new hypotheses were also presented to the reader. The two most popular hypotheses today are about "Cryptic Female Choice" (wherein the male genitalia are thought to be courtship devices) and "Sexually Antagonistic Coevolution" (wherein the male genitalia and female genitalia are constantly coevolving to counteract the opposite sex in an evolutionary "arms race"; p. 49). Each hypothesis provides interesting perspectives for the reader to consider, and from an educational point of view, could be used to stimulate class discussion. Overall, this chapter does a great job at providing current (and past) competing hypotheses in the field.

The chapter by Edward H. Miller on the "Genitalic Traits of Mammals" was extremely intriguing. One interesting aspect in particular was the discussion regarding how testes size is related to mating system (small in single-male breeding systems and large in multi-male mating systems) and how it is most likely associated with the level of sperm competition in a species. This chapter also talks about genitalia as a form of communication in mammals: "sexual 
recognition and mating in mammals entail all senses, and involve components of biochemistry, physiology, behavior, and morphology" (p. 481). Interestingly enough, there is some variation in morphological traits that is supposed to be indicative of age or social rank. Further, this idea can be taken to the extreme when one considers genitalic mimicry or "andromimicry" in some female mammals. Basically, the labia fuse during fetal life to form a "pseudo-scrotum" causing the females to lack an external vagina. A good example given is in female spotted hyaena (Crocuta crocuta), where it is thought that andromimicry likely evolved to reduce interfemale aggression (p. 484). If female genitalia look morphologically different (more similar to a male in this case), this would greatly reduce the likelihood that other female conspecifics would single her out and be aggressive toward her. Overall, this chapter was great, and the authors of this review only had one issue - which is something of an issue we have with this volume writ large - there was no mention of humans. Again, coming from a background in human research psychology, the authors of this review hoped to see a chapter on primary and secondary sex characters in Homo sapiens following up the general chapter on mammals, but to our dismay, it didn't exist. In general, allusions and applications to the world of human mating were absent. It would seem that such an extensive book on the evolution of primary sexual characters in animals would include one of the most interesting animals: ourselves. Granted, the authors of this review are being nit-picky; overall, we would highly recommend this book, but perhaps, we can see an addition of a chapter on humans as a possible improvement in a potential future edition.
Taken as a whole, this book is a must-read. The introductory chapters were filled with plenty of useful information that could be used to teach college students evolutionary theory. Many of the essays on assorted animals were fascinating to say the least, and the variation of creatures was extremely impressive. Overall, the book provided the reader with a cohesive body of knowledge on many species' genitalia and the evolutionary forces that shaped the morphology of sexual characters in these species. The use of examples (diagrams, pictures, etc.) throughout the book was an excellent addition, and truly pulled the readers interest inward. The only issue with the book as a whole was that there was no mention of how evolution shaped human genitalia, but this complaint is dwarfed by the benefits that one could get out of reading this book. The Evolution of Primary Sexual Characters in Animals is lengthy (537 pages), but if taken in stride, is an easy, educational read. That being said, one can only imagine all the information that was not included in this review due to brevity.

The Evolutionary Studies Consortium is founded on the premise that evolution applies to humans along with the rest of life. Leonard and Córdoba-Aguilar's collection here provides an outstanding review of the current data we have on the evolution of sexual characteristics within the animal kingdom. But biologists should embrace the applications of evolution to our own kind - and future scholarship in this area would have even more educational value if such applications were addressed more seriously in this thread of work. That said, this book provides an outstanding collection of work on evolution applied to the nature of sex-anyone who seriously pores through the pages of this book will essentially have a deep appreciation for the evolution of life as we know it. 